to be active in the College through the Collegiate Trainees' Committee (CTC), pointing out how lucky psychiatrists are as they are unique among the Royal Colleges in having trainee representation. He saw the CTC both as generating ideas for the College and as a source of thought-provoking criticism.

Next came Professor W. Parry-Jones (Professor in Child \& Adolescent Psychiatry, Glasgow) who gave quite a meaty discourse on 'Serendipity in Subspecialty Selection', expressing his concern that too much career choice within psychiatry was left to it. In the past trainees moved easily between different areas of psychiatry, but nowadays increasing subspecialisation, although producing increasing expertise, is resulting in fragmentation of the profession and therefore of care. He worried that on current rotational training schemes it was often the "luck of the draw" which sub-specialities a trainee is exposed to. He felt that research was very important and was concerned that in his own sub-speciality there is currently a predominance of those who are "practice and technique orientated" rather than "academic". Overall, he recommended maintaining a broadbased training; increasing marketing of the subspecialities; modification of training to individual needs with more informed career guidance; compulsory training in research; and encouragement of the "less parochial, more mobile" trainee. His thoughts generated some heated argument - much of it over lunch.

Dr Dinesh Bhugra (Chairman, CTC, 1989-1990) then gave a useful and much appreciated account of the structure and function of the CTC. He continued Professor Sim's theme, this time focusing on the CTC, and gave an interesting audit of its activities over the last year leaving us with a clear insight into its important role in psychiatric training. He urged us to continue to be, or start to be, militant.

The "aperitif before lunch" was given by $\mathrm{Dr}$ D. Coia (Consultant Psychiatrist, Glasgow), who talked entertainingly on 'Perspectives of Mental Illness' - the title of an art exhibition running at her Psychiatric Day Hospital. She told how this piece of
Glasgow culture had been most effective in its aim of "encouraging the public to think of mental illness and its effects" on relatives and staff as well as the patient, and it had been a pleasing way of questioning the stigma against mental illness. She showed slides of the varied, beautiful and moving exhibits and quoted from correspondence from exhibitors, one of the best received being "when I was mad I lived in London".

The afternoon talks were slightly more informal. Dr C. Freeman (Consultant Psychiatrist, Edinburgh) talked on 'Planning and Surviving Your First Research Project' and presented much useful information in a humorous and enthusiastic way. He encouraged trainees that you do not have to be born a researcher and that by learning a few tricks of the trade, almost anyone can have a go. He felt a good title was imperative and suggested randomly/freely associating "buzz words" to come up with an eyecatching one. He discussed how to dream up an idea for research, for a literature search, construct a precise hypothesis, design a study, fund raise, get supervision and cope with failure.

Dr A. Hughes (Consultant Psychiatrist, Paisley) ended the day with a talk entitled 'Letting Others Know' about publishing and presenting your research. At the final feedback session this seemed to have been appreciated as a rare opportunity to hear from a skilled communicator 'how to do it'. His talk gave much useful, practical information on presentation as well as advice on how to survive the ordeal of preparing and giving papers and talks. He recommended talks as having the advantages of being forgotten and if talking abroad, it possibly being assumed that you are an expert by virtue of having travelled that far. A written paper on the other hand is harder to disown, although usually better for the CV.

Overall, trainees seemed to feel that the day had been a success and provided a reasonable balance of information, ideas and entertainment. Next year the venue is Edinburgh - not the City of Culture.

\title{
General Hospital Psychiatry
}

Elsevier have agreed to offer the College a $50 \%$ discount subscription rate to General Hospital Psychiatry provided that there are 50 members of the College who subscribe.

The usual 1991 personal subscription rate is US $\$ 98$, the $50 \%$ discount would be $\$ 49$ plus an additional charge of $\$ 26$ for air postage and handling for each subscription. Thus for $\$ 75$ (about $£ 42$ ) you would receive the Journal bi-monthly. This rate is for personal subscription only and not for libraries. However, if there was one nominated psychiatrist at each centre who took General Hospital Psychiatry this would greatly increase the availability of the important articles in the Journal.

If you are interested, please contact Mrs Jean Wales, Royal College of Psychiatrists, 17 Belgrave Square, London SW1X 8PG. 